\title{
Evaluación de la Carga de Enfermedad por el Virus del Papiloma Humano en Bogotá
}

\section{Evaluating the burden of disease caused by human papillomavirus in Bogota}

\author{
Fernando de la Hoz-Restrepo ${ }^{1}$, Nelson Alvis-Guzmán² ${ }^{2}$ Javier Narváez ${ }^{1}$ y
} Luz A. Chocontá-Piraquive ${ }^{1}$

\footnotetext{
1 Departamento de Salud Pública, Facultad de Medicina. Universidad Nacional de Colombia. Bogotá.fpdelahozr@unal.edu.co,janarvaezg@unal.edu.co, lachocontap@unal.edu.co

2 Facultad de Ciencias Económicas, Universidad de Cartagena, Colombia. nalvis@yahoo.com
}

Recibido 6 Octubre 2008/Enviado para Modificación 10 Abril 2009/Aceptado 12 Mayo 2009

\section{RESUMEN}

Objetivo Se realizó un estudio en Bogotá para estimar la magnitud de la carga de enfermedad asociada a la infección por el virus del papiloma humano (VPH) y la proporción potencialmente evitable con el uso de vacunas.

Materiales y métodos Se combinaron una revisión sistemática de la literatura colombiana sobre infección por VPH, el análisis de datos rutinarios de vigilancia de Bogotá y un análisis de costos de la enfermedad.

Resultados Después de corregir por subregistro y mala clasificación en Bogotá se registrarían 322 muertes y 676 casos asociados a cáncer de cuello uterino anualmente. Esto ocasiona la pérdida de 1,5 años de vida por cada 1000 mujeres al año, la mayoría en mujeres entre los 40 y 69 años. Adicionalmente, se diagnosticarían 6 084 lesiones de cuello uterino de alto grado y 22984 de bajo grado. El costo de la enfermedad ascendería a 7 millones de dólares anuales aproximadamente. Se encontraron debilidades en el manejo de los casos con displasias o neoplasias cervicales.

Conclusión El fortalecimiento de los programas de prevención de cáncer de cuello uterino en Bogotá permitiría disminuir de manera importante el impacto epidemiológico y económico de la infección por VPH en Bogotá. Se podría considerar, adicionalmente, la inclusión de una vacuna contra VPH pero solo incluida dentro de una estrategia integral de control y a un precio que pueda ser sostenible.

Palabras Clave: Infecciones por Papillomavirus, vacuna, costo de enfermedad, Colombia (fuente: DeCS, BIREME)

\section{ABSTRACT}

Objective A study was carried out in Bogota aimed at estimating the burden of disease associated with human papillomavirus infection (HPV) and the potentially avoidable percentage due to using new vaccines.

Methods A literature review was combined with analysing surveillance system data and disease cost evaluation. 
Results After adjusting for underreporting and misclassification, it was estimated that 322 deaths from cervical cancer occur annually in Bogota (corresponding to 676 new cases). This would cause the loss of 15 years of life for each 1,000 women per year (most occurring amongst women aged 40 to 69). In addition to cervical cancer, there would be around 6,084 cases of high-grade and 22,984 low-grade cenvical lesions yearly. The disease's yearly cost would amount to around 7 million dollars. Important weaknesses were found in the clinical management of women suffering from cervical lesions.

Conclusion Strengthening cervical cancer prevention programmes in Bogota would lead to saving a significant number of deaths, cases of cancer and the costs associated with HPV infection. Introducing an anti-HPV vaccine may be considered, but only as part of a more widespread preventative strategy and provided that more affordable prices have been found.

Key Words: Papillomavirus infection, vaccine, cost of illness, Colombia (source: $\mathrm{MeSH}$, NLM)

$\mathrm{E}$ $1 \mathrm{VPH}$ es un virus ADN de doble cadena perteneciente a la familia Papovaviridae. Los papilomavirus fueron descritos por primera vez en 1907 por Giuseppe Ciufo quien descubrió la naturaleza infecciosa de las verrugas o papilomas, sin embargo no fueron estudiados hasta los años 70 , cuando apareció la virología molecular y zur Hausen inició los estudios acerca de la génesis infecciosa del cáncer de cérvix (1). La infección por el VPH constituye la ITS más común entre las mujeres (2). Se estima que alrededor de 291 millones de mujeres son portadoras. De éstas, 105 millones están infectadas por los VPH 16 y 18. La prevalencia de la infección es máxima entre las mujeres jóvenes, declinando en mujeres de edad mediana, hasta los 65 años donde se presenta un nuevo pico de infección, los motivos de este segundo pico aún no han sido aclarados (3).

Actualmente existe gran cantidad de evidencia epidemiológica que asocia el VPH al cáncer de cuello uterino (CCU). El estudio de casos de cáncer de cérvix más grande fue llevado a cabo por la IARC, participaron alrededor de 1.000 mujeres en 22 países del mundo y se detectó ADN viral en $97,4 \%$ de los casos, lo cual permitió establecer que la infección por el VPH constituye una causa necesaria, aunque no suficiente, para el desarrollo de esta neoplasia (4).

Para el 2002 se estimaron a nivel mundial 493000 casos nuevos de CCU y 273505 muertes por esta causa, más del $80 \%$ de esta carga pertenece a los países menos desarrollados. En la región de las Américas se produjeron 80253 casos de CCU y 35322 muertes, de los cuales el 81,7 y el 83,6\%, respectivamente, ocurrieron en Centro y Suramérica (5). Esta alta morbimortalidad y el 
hecho de que existen medidas tanto preventivas como terapéuticas para controlar el cáncer de cérvix es lo que urge la puesta en práctica de intervenciones efectivas ya conocidas, así como la evaluación de nuevas intervenciones.

Recientemente se aprobaron dos vacunas contra el VPH producidas por Merck y Glaxo Smith Kline. Una bivalente contra los tipos 16 y 18 y otra cuadrivalente que incluye además los genotipos 6 y 11. Estas vacunas indu-cen anticuerpos contra la principal proteína de la cápside viral, la proteína L1 y se sabe que inducen una respuesta inmune 10 veces mayor a la de la infección natural durante al menos 48 meses (6). Los estudios de campo han mostrado que las vacunas tienen casi $100 \%$ de eficacia contra las lesiones de alto grado producidas por los virus 16 y 18 , pero debido a la historia natural de la enfermedad aún no se ha podido demostrar en qué grado reduce la frecuencia de cáncer inducida por estos virus. Aunque los beneficios potenciales de la vacuna generen expectativa, el uso que de ésta pueda hacerse en programas sistemáticos de inmunización aún no ha sido definido con claridad en Colombia y Latinoamérica debido parcialmente a los altos costos de la vacuna y a la necesidad de realizar estudios de carga de enfermedad que aclaren cuál es el impacto de la infección por VPH entre nosotros y cuál sería el impacto potencial de las vacunas.

En la búsqueda de respuestas a este problema, la Secretaría de Salud de Bogotá y la Universidad Nacional de Colombia, desarrollaron un proyecto de estimación de la carga de enfermedad por Virus del Papiloma Humano en Bogotá, para apoyar la toma de decisiones con relación a la introducción de estas vacunas.

\section{METODOLOGÍA}

El estudio se llevó a cabo en dos fases: a) Estimación de la carga de enfermedad, para lo cual se realizó una revisión sistemática de la literatura colombiana sobre infección por VPH, revisión de la literatura gris, consulta a expertos para validar datos epidemiológicos y un análisis de datos secundarios provenientes de fuentes de información rutinaria de la Secretaría de Salud de Bogotá y b) Estimación del impacto económico de la carga de enfermedad por VPH, mediante una descripción de los costos de la misma. 
Revisión de la literatura: los artículos científicos se buscaron en las bases de Medline, Scielo y LILACS con los siguientes términos Mesh: Uterine Cervical Neoplasms OR Uterine Cervical Dysplasia OR Cervical Intraepithelial Neoplasia OR Papillomavirus Infections OR Alphapapillomavirus AND Prevalence OR Incidence OR Epidemiology AND Colombia. Inicialmente se recuperaron los resúmenes de los artículos identificados con los buscadores descritos. Estos resúmenes se revisaron para determinar cuales tenían información epidemiológica de incidencia, prevalencia y/o mortalidad en Colombia que justificara la lectura del texto completo. Se excluyeron los artículos sobre VPH donde el abordaje fue biomédico o molecular, aquellos que utilizaron Captura Híbrida II para buscar VPH en lesiones y aquellos que no presentaban datos originales (revisiones).

Los desenlaces de interés para la estimación de la carga de enfermedad corresponden a la infección por VPH sin lesión, el cáncer de cuello uterino y lesiones premalignas asociadas a la infección por el VPH, y su definición es la dada por la Clasificación Internacional de Enfermedades, décima versión (CIE 10).

Análisis de Incidencia: Se estimó el número de casos incidentes anuales en Bogotá con base en las estimaciones publicadas por el Instituto Nacional de Cancerología INC- y los registros de mortalidad de Bogotá. También se calcularon los casos anuales por grupo de edad de acuerdo con los datos del Registro Poblacional de Cáncer de Cali (RPCC). La incidencia publicada se validó usando los datos de mortalidad y la relación caso-muerte para el cáncer de cuello uterino.

Análisis de Mortalidad: Se realizaron estimaciones de la mortalidad a partir de las tasas publicadas por el INC y la International Agency for Research on Cancer -IARC- y se analizaron los registros de mortalidad recolectados por la SDS de Bogotá por cáncer de cuello uterino y por otros cánceres relacio-nados con el VPH de 1998 a 2006. Las estimaciones de mortalidad por CCU basadas en la literatura fueron validadas usando los registros de mortalidad extraídos de los certificados de defunción para los años 2003, 2004 y 2005, suplidos por la Secretaría de Salud de Bogotá (7). Complementariamente se usaron los datos de series y proyecciones de población del DANE para estimar la población de mujeres residente en Bogotá entre los años 2002 y 2006 (8). Con esta información se proyectó la población a 1 de enero de 2003 y a 31 de diciembre de 2005. Además se utilizó el método de Bennett-Horiuchi (9) para estimar el grado de subregistro de la mortalidad usando la información descrita 
anteriormente. Los registros de mortalidad fueron corregidos luego de estimado el subregistro y la mala clasificación. Una fracción importante de las muertes por cáncer de útero se reportan sin localización específica (código CIE10 C55X Tumor maligno del útero, parte no especificada) lo cual no permite identificar si se trata de cáncer de cuello o de cuerpo; por otra parte, una proporción de las muertes que se registran como debidas al cáncer de cuerpo uterino son en realidad cánceres de cuello.

Para corregir los dos problemas mencionados se utilizó una modificación del algoritmo propuesto por el estudio de Carga de la Enfermedad en Colombia (10) para la reclasificación de la mortalidad por cáncer del útero, de la siguiente manera:

Muertes por cáncer de cuello uterino=Número de muertes por cáncer de cuello uterino registradas $+\alpha^{*}$ Muertes por cáncer de útero de lugar no especificado $+\beta^{*}$ Muertes por cáncer de cuerpo de útero registradas.

Para los tres años estudiados se usó á=0,9 (es decir, se consideró que el $90 \%$ de las muertes por cáncer de útero de sitio no especificado correspondían a cánceres de cuello y el $10 \%$ a cuerpo), y $\beta$ variando entre 0,3 y 0,5 dependiendo del grado de sobreestimación de la mortalidad por cáncer de cuerpo en cada año.

Además, para la estimación de la carga de enfermedad se calcularon los Años Potenciales de Vida Perdidos -APVP-

Análisis de costos. Para el análisis de costos de la carga de enfermedad se tomaron los costos de referencia usados para Colombia en el estudio del Instituto Sabin (11). En este estudio los costos están estimados en dólares internacionales del 2005. Dado que se trata de costos medios anuales de manejo de casos, los costos de la carga de enfermedad se estimaron para un año. El número de casos corresponde a los estimados a partir del análisis de bases de datos de colposcopia y de la mortalidad de Bogotá, corregidos por subregistro y relación muerte: caso de 1:2,1 (676 casos anuales). El número de lesiones de bajo grado se calculó con base en el diagnóstico histológico. 


\section{RESULTADOS}

Revisión sistemática

Se obtuvieron 26 artículos que respondían a los términos de búsqueda y luego de revisar los resúmenes se escogieron 13 para su lectura en texto completo.

Prevalencia de la Infección por VPH

Se encontraron 4 estudios de prevalencia de infección en mujeres normales y con diferentes tipos de lesión. Los estudios de Bogotá eran encuestas de prevalencia mientras que los de Cauca y Cali eran de casos y controles. La prevalencia de VPH de alto riesgo en lesiones de alto grado y cáncer estaba entre 70 y $90 \%$, mientras que la frecuencia de virus 16 y 18 en cánceres estaba entre 52 y $64 \%$. Los resultados completos se describen en la Tabla 1. Con la distribución por edad de las mujeres de Bogotá en 2005, se esperarían 350000 mujeres infectadas con cualquier tipo de VPH, de las cuales el $74 \%$ tendría una infección por un virus de alto riesgo (Tabla 2).

Tabla 1. Prevalencia de infección por VPH según tipo de lesión en diferentes estudios realizados en Colombia

\begin{tabular}{|c|c|c|c|c|c|}
\hline \multirow[b]{2}{*}{ Diagnóstico } & \multirow[b]{2}{*}{$\mathrm{N}^{\circ}$} & \multicolumn{4}{|c|}{ VPH (\%) } \\
\hline & & $\begin{array}{c}\text { Alto } \\
\text { Riesgo }\end{array}$ & $\begin{array}{c}\text { Bajo } \\
\text { Riesgo }\end{array}$ & $\begin{array}{c}\text { Cualquier } \\
\text { Tipo }\end{array}$ & $\begin{array}{l}\text { VPH } \\
16 / 18^{*}\end{array}$ \\
\hline \multicolumn{6}{|l|}{ BOGOTA (17) } \\
\hline Normal & 1845 & 11,4 & 3,2 & 14,9 & \\
\hline ASCUS/AGUS & 44 & 4,6 & 4,6 & 9,1 & \\
\hline LEI Bajo Grado & 70 & 47,1 & 4,3 & 55,7 & \\
\hline LEI Alto Grado/ Cáncer & 10 & 70 & 10 & 80 & \\
\hline \multicolumn{6}{|l|}{ CAUCA (12) } \\
\hline NIC II/III y Ca in Situ & & 91 & & & \\
\hline \multicolumn{6}{|l|}{ CALI (13) } \\
\hline NIC III/ Ca in Situ & 125 & & & 63,2 & 51,9 \\
\hline \multicolumn{6}{|l|}{ BOGOTA (17) } \\
\hline Sin displasia ${ }^{\dagger^{\prime}}$ & 115 & & & $21,7^{\ddagger}$ & 50,0 \\
\hline NIC I & 55 & & & 49 & 44,4 \\
\hline NIC II & 37 & & & 37,8 & 85,5 \\
\hline NIC III/ Ca in Situ & 20 & & & 55 & 63,6 \\
\hline
\end{tabular}


Tabla 2. Prevalencia de infección por VPH por grupo etáreo en mujeres con citología normal y estimación de la carga de infección para la población de Bogotá en el 2005

\begin{tabular}{lcccccc}
\hline Edad & $\begin{array}{c}\text { Cualquie } \\
r\end{array}$ & $\begin{array}{c}\text { Prevalencia (17) } \\
\text { VPH Alto } \\
\text { VPH }\end{array}$ & $\begin{array}{c}\text { VPH } \\
\text { Rajo } \\
\text { Riesgo }\end{array}$ & $\begin{array}{c}\text { Cualquier } \\
\text { VPH }\end{array}$ & $\begin{array}{c}\text { VPH Alto } \\
\text { Riesgo }\end{array}$ & $\begin{array}{c}\text { VPH Bajo } \\
\text { Riesgo }\end{array}$ \\
\hline $13-19$ & 26,1 & 20,4 & 5,2 & 74754 & 58428 & 14893 \\
$20-24$ & 22,7 & 18,2 & 4,1 & 76653 & 61457 & 13845 \\
$25-29$ & 12,7 & 10,4 & 1,8 & 40594 & 33243 & 5754 \\
$30-34$ & 16,6 & 11,9 & 4,2 & 46751 & 33514 & 11829 \\
$35-39$ & 8,1 & 6,0 & 2,1 & 22449 & 16629 & 5820 \\
$40-44$ & 8,1 & 7,8 & 0,5 & 21746 & 20941 & 1342 \\
$45-54$ & 2,3 & 2,3 & 0 & 9397 & 9397 & 0 \\
$\geq 55$ & 13,2 & 5,7 & 7,6 & 58815 & 25397 & 33863 \\
\hline Total & & & & 351159 & 259006 & 87346 \\
\hline
\end{tabular}

Mortalidad por Cáncer de Cuello Uterino (CCU)

Entre 1998 y 2002 la Secretaría Distrital de Salud de Bogotá registró en promedio 222 muertes anuales (DE: 20,2) con causa básica de muerte por CCU. La distribución de estas muertes por grupo de edad muestra una baja mortalidad antes de los 25 años, que se incrementa abruptamente hasta alcanzar su pico entre los 45-54 años y después desciende paulatinamente hasta los 75 y más años de edad. El número de muertes se incrementa en $27 \%$ entre 1998 y 2005 , pero la tendencia al alza se interrumpe en 2004. De acuerdo con la IARC (5), las muertes por cáncer de cuerpo uterino deberían representar solamente el 7,3 \% del total de muertes por cáncer del útero en Colombia, pero esta proporción era más elevada en Bogotá, lo cual sugiere que como resultado de la mala clasificación, el número de muertes atribuidas al cáncer de cuerpo uterino podría estar sobreestimado por un factor entre 1,65 y 2,24 durante el periodo estudiado.

Una vez aplicadas las correcciones a los registros de mortalidad, el número estimado de muertes por CCU sería de 362, 314 y 362 para los años 2003,2004 y 2005, respectivamente. Esto correspondería a una tasa promedio de 9,87 por 100000 (IC95 \% 8,9 - 10,98) para todo el periodo observado. Con esta tasa, el número de muertes proyectadas al 2008 sería de 366 . Las muertes por cáncer de cuello uterino generarían 5358 Años potenciales de vida perdidos (APVP) cada año, el $68 \%$ de estos $(3$ 672) se pierden en mujeres entre los 40 a 69 años. La tasa de pérdida de APVP es de 1,5 por cada 1000 mujeres. 
Estimación de la Incidencia de CCU a partir de la mortalidad De acuerdo a los datos del INC la relación de mortalidad e incidencia de cáncer de cuello uterino en Bogotá es de 1:2,1, es decir aproximadamente dos casos de cáncer por cada muerte por lo que para 2008 se esperarían 769 casos ( 2,1 x 366 muertes), es decir la incidencia de cáncer de cuello sería de 20,7 por 100000 mujeres año.

Mortalidad por otros tipos de cáncer relacionados con el VPH Los cánceres de ano, vulva, vagina y pene también se han relacionado con la infección por el Virus del Papiloma Humano. El total de muertes por estos cánceres entre 1998 y 2006 fue de 560, con un promedio de 62 muertes por año. Las muertes por cáncer de boca y orofaringe representaban el $55 \%$.

Tabla 3. Diagnósticos colposcópicos reportados

\begin{tabular}{|c|c|c|c|c|c|}
\hline $\begin{array}{l}\text { Grupo de } \\
\text { edad }\end{array}$ & $\begin{array}{c}\text { ASC (US- } \\
\text { H) (\%) }\end{array}$ & $\begin{array}{c}\text { LEIBG } \\
(\%)\end{array}$ & $\begin{array}{c}\text { LEIAG } \\
(\%)\end{array}$ & $\begin{array}{c}\text { Cáncer } \\
(\%)\end{array}$ & Total $(\%)$ \\
\hline $13-20$ & 0 & $71(53,8)$ & $3(2,3)$ & 0 & $74(56,1)$ \\
\hline $20-24$ & $1(0,3)$ & $\begin{array}{c}188 \\
(50,3)\end{array}$ & $30(8,0)$ & 0 & $\begin{array}{c}219 \\
(58,6)\end{array}$ \\
\hline $25-29$ & $1(0,3)$ & $\begin{array}{c}172 \\
(45,1)\end{array}$ & $39(10,2)$ & $1(0,3)$ & $\begin{array}{c}213 \\
(55,9)\end{array}$ \\
\hline $30-34$ & $1(0,3)$ & $\begin{array}{c}141 \\
(38,3)\end{array}$ & $39(10,6)$ & $1(0,3)$ & $\begin{array}{c}182 \\
(49,5)\end{array}$ \\
\hline $35-39$ & $1(0,3)$ & $\begin{array}{c}143 \\
(35,9)\end{array}$ & $34(8,5)$ & $2(0,5)$ & $\begin{array}{c}180 \\
(45,2)\end{array}$ \\
\hline $40-44$ & $1(0,2)$ & $\begin{array}{c}165 \\
(37,6)\end{array}$ & $39(8,9)$ & $1(0,2)$ & $\begin{array}{c}206 \\
(46,9)\end{array}$ \\
\hline $45-54$ & $1(0,2)$ & $\begin{array}{c}151 \\
(25.3)\end{array}$ & $39(6,5)$ & $3(0,5)$ & $\begin{array}{c}194 \\
(32,5)\end{array}$ \\
\hline $55-86$ & 0 & $25(11,6)$ & $20(9,3)$ & $7(3,2)$ & $52(24,1)$ \\
\hline Total & $6(0,2)$ & $\begin{array}{l}1056 \\
(36,4)\end{array}$ & $243(8,4)$ & $15(0,5)$ & $\begin{array}{l}1320 \\
(45,4)\end{array}$ \\
\hline
\end{tabular}

Frecuencia de otras lesiones cervicales a partir de datos de colposcopias Se analizó la información de 2899 colposcopias de 4 hospitales de Bogotá, la mediana de edad de las participantes era de 38 años, con un rango entre los 13 y los 86 . En el caso de mujeres con más de una colposcopia se escogió la información del primer diagnóstico, en la Tabla 3 se describe la proporción de diagnósticos colposcópicos de lesiones de bajo grado -LEIBG- y alto grado -LEIAG- y cáncer. Se encontró que por cada caso de cáncer diagnosticado por colposcopia se reportaron 70,4 LEIBG y 16,2 LEIAG. Para biopsias, cada caso de cáncer se correspondía con 33,8 casos de LEIBG y 9,06 de LEIAG. Aplicando a los casos de cáncer estimados para Bogotá la 
relación encontrada para las biopsias, obtendríamos que para el 2008 se esperarían, por 769 casos de cáncer nuevos, 6967 casos de lesiones de alto grado y 25992 de bajo grado. Las tasas anuales de ocurrencia de lesiones de alto y bajo grado serían de 188 y 702 casos por 100000 mujeres/año respectivamente.

Tabla 4. Costos en dólares de la carga de enfermedad por cáncer

\begin{tabular}{lccc}
\multicolumn{4}{c}{ de cuello uterino } \\
\hline \multicolumn{1}{c}{ ESTADIO } & $\begin{array}{c}\text { Costo medio/caso } \\
(11)\end{array}$ & Casos $^{* *}$ & $\begin{array}{c}\text { Costos de Carga } \\
\text { Dólares internacionales de 2005 }\end{array}$ \\
\hline NIC I* & 120 & 24000 & 2880000 \\
NIC II/ Ca In Situ & 1373 & 186 & 255705 \\
IA & 6039 & 27 & 161240 \\
IB & 7223 & 105 & 759215 \\
IIA & 7713 & 19 & 143385 \\
IIB & 7895 & 102 & 808559 \\
IIIA- IIIB & 8249 & 184 & 1513972 \\
IVA & 8543 & 17 & 141489 \\
IVB & 8986 & 37 & 331062 \\
\hline Totales & & & 6858426 \\
\hline
\end{tabular}

*Los NIC I corresponden a la estimación usando la relación caso de cáncer: LEIBG confirmada por biopsia de 1:33,8; ** Se asumen 676 casos de cáncer de cuello uterino. Este es el promedio anual de casos esperados para el periodo 2002-2006 corregido por subregistro, mala clasificación y asumiendo un índice caso-muerte de 2:1

Costos de la Carga de Enfermedad por lesiones cervicales

Los resultados en la Tabla 4 muestran los costos directos del manejo de las lesiones precancerosas y del cáncer cervical por estadíos. Asumiendo que la carga de nuevos casos de cáncer cervical cada año es de 676 correspondientes al promedio anual calculado con base en la mortalidad corregida en el periodo 2002 a 2006, y que estos se distribuirían por estadio al momento del diagnóstico de acuerdo con la estadificación del INC, con estos datos los costos de carga de enfermedad para Bogotá estarían alrededor de los 7 millones de dólares por año (Dólares PPA 2005).

\section{DISCUSIÓN}

Los cánceres ligados a la infección por el VPH son un problema importante de salud pública en Bogotá. El CCU, que es de lejos la consecuencia más frecuente de esta infección, mata entre 250 y 350 mujeres cada año en la ciudad, mientras que se producirían entre 500 y 1.000 casos nuevos. Esta cantidad de muertes representaría alrededor del $10 \%$ de todas las muertes reportadas al DANE por CCU en el país. Las muertes por CCU representan entre el 2 y el $3 \%$ de todas las muertes de mujeres mayores de 15 años 
en la ciudad y causan 5000 APVP cada año. Esta proporción varía según la edad, siendo muy alta para el grupo de edad de 35 a 54 años donde el CCU representa alrededor del $6 \%$ de todas las muertes en ese grupo.

Uno de los aspectos más preocupantes de la ocurrencia de mortalidad por CCU en Bogotá es su tendencia al incremento, ya que pasó de una tasa de 6,2 por 100000 mujeres en 1998 a 7,3 en 2003, un aumento de $18 \%$. Durante 2004 se vio una reducción de la mortalidad por esta neoplasia pero ésta fue tan abrupta y de tan corta duración que difícilmente podría atribuírsele a una causa diferente que a problemas en el registro de la mortalidad. No es posible en este análisis establecer cuáles pueden ser las causas de este au-mento, que pueden ir desde una mejoría en los sistemas de registro hasta un aumento real de la mortalidad por esta causa debido a un efecto de cohorte. Sin embargo, hay alguna evidencia de que la calidad del registro para CCU no ha mejorado mucho en los últimos años, como lo muestra la proporción de CCU que no han sido clasificados como causa básica de muerte en los registros del DANE.

Adicionalmente a la mortalidad y la ocurrencia de cáncer, la infección por VPH también contribuye a la carga de enfermedad en Bogotá causando un número apreciable de lesiones preneoplásicas y cambios patológicos en el cuello uterino que ocasionan gastos al sistema de salud, además de ansiedad y pérdida de calidad de vida en aquellas personas en quienes se diagnostican estas lesiones.

Aunque no existe un registro oficial de lesiones de alto y bajo grado en Bogotá ni en el país, intentamos aproximarnos a la magnitud de estos problemas usando algunos registros de colposcopias y biopsias de hospitales de la ciudad. Empleando las relaciones entre número de casos de cáncer de cérvix y las otras lesiones diagnosticadas, estimamos que anualmente en Bogotá se diagnosticarían entre 6000 y 7000 mujeres con lesiones de alto grado y 26 000 a 28000 con lesiones de bajo grado lo cual contribuye de manera sus-tancial al costo de la enfermedad.

La contribución de otros cánceres a la carga de enfermedad por VPH es relativamente baja en Bogotá, como es también para otras partes del mundo y Latinoamérica. Según los registros del DANE para mortalidad en Bogotá, entre 1998 y 2006 se produjo un promedio anual de 7 casos de muerte por cáncer de ano, 8 de cáncer de vulva, 4 de cáncer de vagina y 9 de cáncer de pene. Es decir, los otros canceres anogenitales asociados al VPH solo re- 
presentarían un 6 a $9 \%$ de todas las muertes por canceres relacionados con VPH, proporción que seguramente es similar para otros indicadores epidemiológicos como incidencia o prevalencia, ya que la sobrevida de estos cánceres es similar o más baja que la de cáncer de cuello uterino. De acuerdo al registro de Cali la incidencia de cáncer de vulva y vagina es de 0,9 y 0,8 por 100 000 respectivamente. La incidencia de cáncer de ano es de 1,2 para mujeres y 0,4 para hombres y la incidencia de cáncer de pene es de 1,5 por 100000 .

Existen varias debilidades potenciales de nuestro estudio que vale la pena resaltar. Una tiene que ver con el bajo número de estudios sobre la epidemiologia del VPH y especialmente sobre la prevalencia de VPH en lesiones cervicales y en otros cánceres, lo que hace que nuestros cálculos estén basados en dos estudios solamente, lo que podría no reflejar la variabilidad de la presencia del virus en diferentes poblaciones. Sin embargo, nuestros estimados coinciden con los de los registros nacionales y las estimaciones del INC, lo que sugiere que esta limitación no afecta de manera importante la validez de los hallazgos.

Otra limitación está relacionada con la fuente de los costos de la enfermedad. Estos debieron ser estimados a partir de fuentes internacionales lo que también puede llevar a cuestionamientos sobre su pertinencia para nuestro medio. Sin embargo, los costos que usamos para el cálculo fueron obtenidos de una consulta a especialistas en el tema de manejo de lesiones cervicales de toda América Latina, por lo que nuestros estimados representarían un promedio regional. Esto sería conservador con respecto a los verdaderos costos de Bogotá, donde la población tiene un mayor acceso a servicios de salud con respecto al promedio de la región latinoamericana.

Las vacunas actuales para VPH incluyen la protección solamente para los tipos 16 y 18 que contribuirían con un $60 \%$ de los cánceres de cuello uterino en Colombia y Bogotá $(12,13)$. Con esta composición de la vacuna y una cobertura de vacunación de $90 \%$ la proporción de canceres cervicales y muertes que podrían evitarse con su uso sería de 50 a $55 \%$. Trasladado a números, esto significaría alrededor de 300 casos y 150 muertes menos por CCU por año, lo cual sería un impacto importante. Esto implica que la introducción de la vacunación sola no contendría el problema del CCU en la ciudad, sino que habría que mantener y aun fortalecer el programa de tamizaje y tratamiento.

Se han realizado varios estudios en América Latina para estimar el costo efectividad de la vacunación contra VPH. En uno muy reciente, realizado bajo 
la coordinación del Instituto Sabin de Washington (11), se encontró que para 6 países de Latinoamérica, incluyendo Colombia, un precio de esquema por encima de 60 dólares americanos (US \$ 20 por dosis) no era costo efectivo, y que por encima de ese valor era mejor usar solo el tamizaje como medida de prevención del cáncer cuello uterino. Dado que Bogotá tiene una tasa de mortalidad por cáncer de cérvix inferior al promedio latinoamericano y colombiano, este valor umbral de costo efectividad (20 dólares la dosis) puede aun ser inferior. En otro estudio, realizado para el escenario de Brasil $(11,14)$ Goldie et al encontraron que a un costo de US $\$ 75$ por mujer vacunada el índice de costo efectividad incremental era de US \$ 1100 por año de vida salvado, mientras que a 100 dólares por mujer vacunada este índice aumentaba a US \$1900, colocándose en el borde de lo que sería muy costo efectivo para Bogotá. Sin embargo, el costo efectividad es solo uno de los criterios a tenerse en cuenta para la toma de decisiones en salud, y cuando el costo de vacunar supera ciertos límites sin generar ahorros netos, es necesario encontrar fuentes de financiación adicionales que ordinariamente no están disponibles y deben ser creadas por los organismos legisladores o tomadores de decisión (14).

Bogotá tiene alrededor de 300000 niñas en edades de 10 a 14 años que serían el blanco de la primera onda de vacunación y luego habría que vacunar alrededor de 70000 niñas cada año, a medida que llegan a los 10 años. Con un precio de mujer vacunada de 300 dólares, esto implica que el presupuesto para vacunar contra VPH en el primer año debe ser de US \$90000 000 y luego de US \$20000 000 anuales, lo cual es sustancialmente mayor que los cerca de US \$33000 000 que gasta el país anualmente en vacunar a toda la población blanco del Programa Ampliado de Inmunizaciones, y más de 10 veces superior al presupuesto de Bogotá para el PAI (aproximadamente USD \$ 6000 000). Indudablemente a esos precios actuales no sería factible ni sostenible la introducción, por lo que hay que considerar la necesidad de encontrar un precio que sea justo para los productores y factible para la ciudad.

Por otra parte, la proporción global de mujeres (18 a 69 años) que se hacen la citología en Bogotá según la encuesta de Demografía y Salud es de 89,2 \%, pero cuando se estratifica por la frecuencia del tamizaje se encuentra que el $54 \%$ menciona hacérsela una vez al año, el 5,6 \% cada dos años y rara vez $18 \%$. Una lectura cuidadosa a estos datos sugeriría que hasta un $40 \%$ de la población femenina en Bogotá no se realiza la citología o lo hace de manera errática, lo cual resalta la necesidad de mejorar el acceso de las mujeres a la información sobre la prueba y a la prueba misma (15). 
La otra parte de la lucha contra el cáncer de cuello uterino tiene que ver con el manejo que se hace de las mujeres con resultados anormales en su citología. En los datos obtenidos de los reportes de colposcopias se observó por ejemplo que mientras en uno de los hospitales casi el $90 \%$ de las LEIBG (diagnosticadas por biopsia) se dejaron en observación, en otro hospital el $69 \%$ se trataron con cauterización o conización, incluso en adolescentes y mujeres jóvenes que no habían sido diagnosticadas con lesión de alto grado ni en la citología ni en la colposcopia. Inicialmente, esta situación podría generar dudas acerca de la calidad de los datos, no obstante, una posible explicación aún más relevante es la deficiente aplicación de los protocolos de manejo mundialmente aceptados, pues se evidencia una alta variabilidad en el manejo incluso al interior de un mismo centro de atención. El objetivo del tamizaje no es solo el tratamiento de las lesiones sino el manejo apropiado de las mismas, especialmente cuando se practican procesos ablativos en mujeres jóvenes, aun sabiendo que estos elevan el riesgo de parto pre término, bajo peso al nacer y ruptura prematura de membranas (16). Hacer claridad sobre estas posibles fallas en la atención y sus razones es de vital importancia.

Finalmente, debe tenerse en cuenta que para la introducción de la vacuna contra VPH se debe reorganizar el PAI y adecuarlo en función de un grupo de edad que no es el blanco tradicional de las programas de vacunación y en función de una vacuna que puede generar cierta controversia en los padres de familia por la característica de la infección que se previene, una ITS

Agradecimientos. El presente estudiofue financiado por la Secretaría de Salud de Bogota. Agradecemos el apoyo de las licenciadas María Patricia González, María Teresa Espitia, Patricia Arce, Sonia Salamanca y del Dr. Osvaldo Cáliz.

\section{REFERENCIAS}

1. IARC. IARC Monographs on the Evaluation of Carcinogenic Risks to Humans. VOLUME 90. Human Papillomaviruses. Lyon: World Health Organization, International Agency for Research on Cancer; 2007.

2. Aral SO, Holmes KK. Social and behavioral determinants of epidemiology of STDs: industria-lized and developing countries. En: Holmes K, Mardh PA, Sparling PF, et al. Sexually Trans-mitted Diseases. New York: McGraw-Hill, 1999; 39-76.

3. Burchell AN, Winer RL, de Sanjose S, Franco EL. Chapter 6: Epidemiology and transmission dynamics of genital HPV infection. Vaccine. 2006 Aug 21;24 Suppl 3:S52-61.

4. Bosch FX, Manos MM, Muñoz N, Sherman M, Jansen AM, Peto J, et al. Prevalence of hu-man papillomavirus in cervical cancer: a worldwide perspective. International biological study on cervical cancer (IBSCC) Study Group. J Natl Cancer Inst 1995; 87(11): 796-802.

5. Globocan, Globocan 2002: Cancer Incidence, Mortality and Prevalence Worldwide. 2004, IARC Lyon. 
6. Stanley M, Lowy D, Frazer I. Vacunas profilácticas contra el VPH. Vaccine, 2006; 24(S3): 113121.

7. Secretaría Distrital de Salud. Registro de Mortalidad de Bogotá 1998 a 2006. 2008, Alcaldía Mayor Bogotá D.C.

8. DANE, Colombia. Estimaciones 1985-2005 y proyecciones $2006-2020$ anualizadas por sexo y edad. 2008.

9. Mathers C, Vos T, Lopez AD, Salomon J, Ezzati M (Ed.). National Burden of Disease Studies: a practical guide: Global Program on Evidence for Health Policy. World Health Organization: Geneva, 2001.

10. Rodríguez J G. H., Mortalidad y años de vida ajustados por discapacidad como medidas de la carga de enfermedad. Colombia 1985 - 1995. 1999, Bogotá D.C.: Ministerio de la Protección Social, República de Colombia.

11. Instituto Sabin, CDC, OPS. [Internet] Carga de enfermedad por el Virus del VPH en Latino-américa y el Caribe y costo efectividad de la vacunación, en I Reunión Latinoamericana sobre control de cáncer de cuello cérvico-uterino. 2008: México DF. Disponible en http:// www.sabin.org/files/dagna_o._constenla.pdf Consultado: Septiembre 2008.

12. Sierra $\mathrm{CH}$, Acosta MP, Orejuela L. Papilomavirus y Factores asociados a Neoplasia Intrae-pitelial Cervical de Alto Grado en Cauca, Colombia. Rev. Salud Publica (Bogotá) 2006; Sup. 8 (1): 47-58.

13. Bosch FX, Muñoz N, de Sanjosé S, Navarro C, Moreo P, Ascunce N, et al. Human papillo-mavirus and cervical intraepithelial neoplasia grade III/carcinoma in situ: a case-control study in Spain and Colombia. Cancer Epidemiology, Biomarkers \& Prevention 1993; 2(5): 415- 422.

14. Goldie SJ, Kim JJ, Kobus K, Goldhaber-Fiebert J, Salomon J, O'Shea M, et al. Cost-effectiveness of HPV 16, 18 vaccination in Brazil. Vaccine 2007; 25(33): 6257-70.

15. Ojeda G, Ordóñez M, Ochoa L, Samper B. Salud sexual y reproductiva: Resultados En-cuesta Nacional de Demografía y Salud 2005. : Asociación Probienestar de la Familia Colom-biana (Profamilia). Profamilia, Bienestar Familiar, United States Agency for Internacional Deve-lopment, Ministerio de la Protección Social; 2005.

16. Wright TC, Massad LS, Dunton CJ, Spitzer M, Wilkinson EJ, Solomon D. 2006 consensus guidelines for the management of women with cervical intraepithelial neoplasia or adenocarci-noma in situ. American Society for Colposcopy and Cervical Pathologysponsored Consensus. American Journal of Obstetrics \& Ginecology Conference 2007; 197(4): 340-345.

17. Molano M, van den Brule AJC, Posso H, Weiderpass E, Ronderos M, Franceschi S, et al. Low grade squamous intra-epithelial lesions and human papillomavirus infection in Colombian women. British Journal of Cancer 2002; 87(12): 1417-1121.

18. Urdaneta $\mathrm{H}$, Calderon $\mathrm{O}$, Amortegui $\mathrm{A}$. Prevalencia de los genotipos del virus del papiloma humano en neoplasia intraepitelial cervical y cancer cervical en Bogota. Rev.colomb.obstet.ginecol 1994; 45(3):219-224.

19. Molano M, Posso H, Weiderpass E, van den Brule AJC, Ronderos M, Franceschi S et al. Prevalence and determinants of HPV infection among Colombian women with normal cytology. British Journal of Cancer 2002; 87(3): 324-333. 